\title{
The impact of the forecasting capacity of one science on that of other sciences
}

\author{
Jan TINBERGEN \\ Emeritus Professor of Economics, Erasmus University, Rotterdam, The Netherlands
}

\section{Introductory}

I am pleased and grateful for the opportunity to present here some remarks on the interrelationships of the forecasting capacity of sciences. I hope that my remarks may be considered a useful comment on forecasting; I don't claim any originality, though. Others have worked on this subject more than I have: see, e.g., Intriligator (1988), Nordhaus (1987), Theil $(1958,1966)$, to mention a few.

\section{Defining elementary scientific areas and group- ing them}

The total of our scientific knowledge and understanding may be ordered with the aid of a number of criteria, such as the time to which it applies, the object it deals with and many other criteria. This ordering may be illustrated graphically in a space with as many dimensions as there are criteria. This can best be understood if we use only two criteria. Then the space is a plane and can be represented by a geographical map of sciences. The most detailed presentation uses a large number of areas (such as municipalities in a geographical map) and these can be grouped into larger areas (provinces) or still larger (nations). This grouping is useful for surveys.

For the discussion of our subject it is desirable to subdivide the space of science (in the widest sense) into elementary areas or micro-sciences, in as logical a way as possible. What we call a science (physics, chemistry, economics, etc.) constitutes in fact a large number of micro sciences or theories (the theory of light, the theory of sound, of gravity; the theory of the labour market, of the balance of payments, etc.). As a consequence what we call a science had better be called a macroscience.

Generally speaking this subject of 'science geography' deserves a more systematic treatment than is usual. An example or common practice is given in table 1 , which is used by the Royal Dutch Academy of Sciences. A systematic subdivision must satisfy the well-known conditions that the individual elementary areas

(i) are circumscribed sufficiently precisely in order to avoid misunderstanding,

(ii) do not overlap, and hence exclude each other, and

(iii) together cover the complete space of scientific activities.

It is clear that table 1 does not satisfy these conditions. It is confusing that biology is not found under the group of life sciences. The presence of dental medicine raises the question where eye or ear medicine can be found. Astronomy is not mentioned anywhere. Literally speaking dental medicine overlaps with medical science. The answer is that the subdivision offered in this table is the result of a process of historical growth and tradition. It seems worth while, however, to make an attempt to use more accurate descriptions. In order to arrive at a really systematic subdivision the following distinctions must be made and this list is not complete, but only meant as a list of examples.

(1) methodological versus object sciences,

(2) theoretical versus applied sciences,

(3) sciences with living and non-living (dead) objects, 
Table 1

Composition of the five spaces of sciences (Royal Dutch Academy of Sciences).

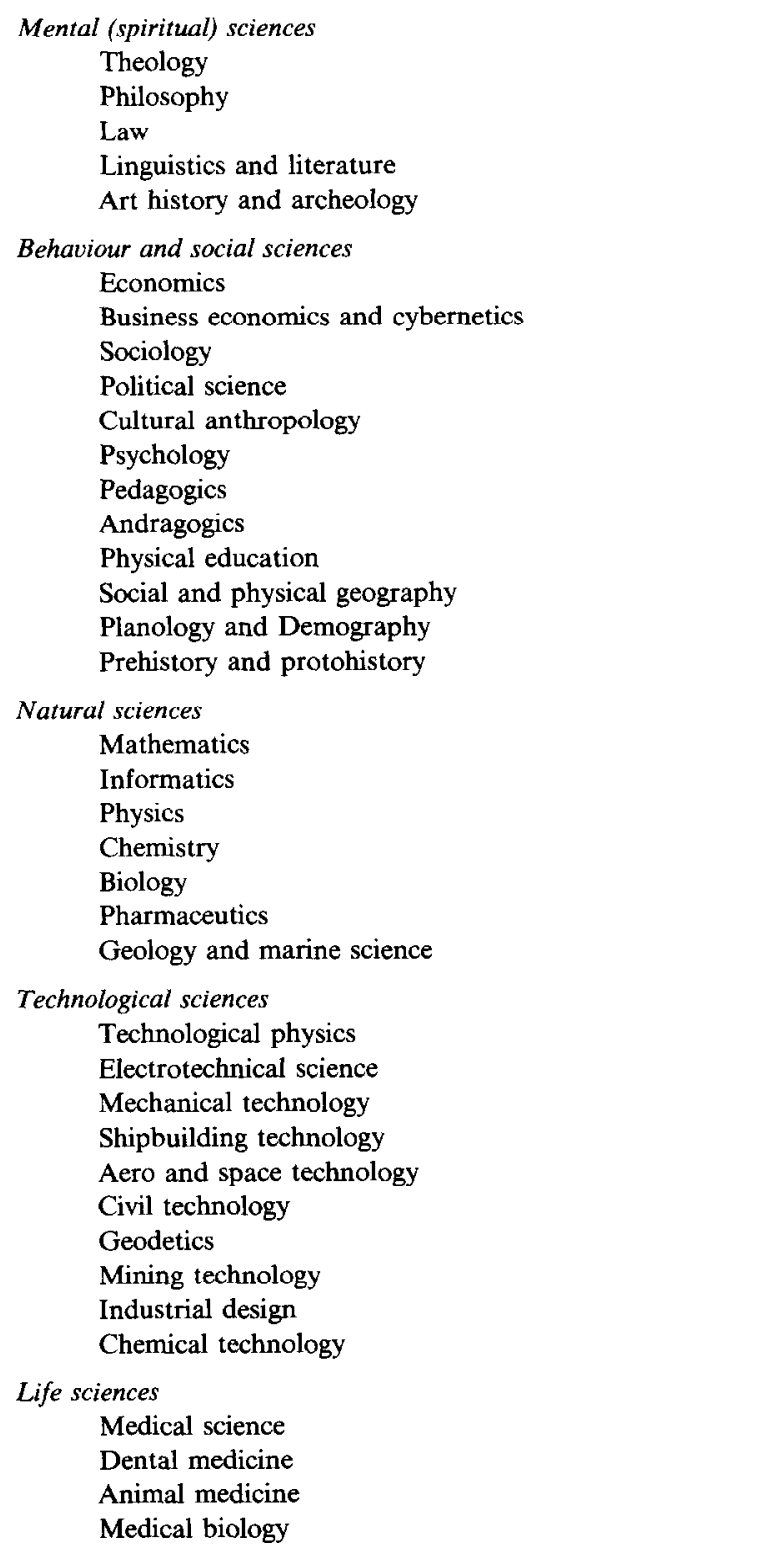

(4) sciences with individual behaviour versus those with group behaviour as their object,

(5) sciences with physical versus those with spiritual behaviour as their object,

(6) ordering of the object of sciences according to a time scale (pre-historic, historic, present, future or a more refined scale).
Some additional information may help to clarify these examples. Methodological sciences are philosophy, algebra, analysis and informatics; sciences with an object are geometry (with space as its object), physics (with the non-living nature), zoology (with animals as its object), etc. The distinction (2) is meant to distinguish pure sciences from technological ones. Distinction (5) is meant to separate sciences about the activities of the body from those about the activities of the brain. My suggestions have to be corrected by the experts of each science, since I am (at most) an expert in a few sciences only. Each member of the 'list' just given constitutes one dimension of the total space of all sciences. The range of each dimension may be subdivided into two criteria only (as (1), (2), (3) and (5)) or more ((4) and (6)). Group behaviour is studied by sociology, but also by pedagogics and the difference may be that all group members are in the same position in sociology, but in a teacher-pupil relation in pedagogics.

\section{Forecasting capacity or efficiency}

One task of a science is to make forecasts, that is calculating the future value of some key variable occurring in that science with the aid of a relation in which that variable is expressed in terms of other variables whose values are known. This is not the only task of a science. Another task may be to find the optimal value of some variable. The Amsterdam Symposium and the International Journal of Forecasting deal with forecasting, however, and here an important issue is whether a forecast is or is not exact or reliable. Some sciences, for instance astronomy of the position of the planets, are extremely reliable and others, such as meterologic forecasts are much less exact. Therefore we may introduce the concept of a science's capability or efficiency of forecasting; see, e.g., Nordhaus (1987).

\section{Forecasting capacity and the interdependence of sciences}

Sciences also show large differences in interdependence with other micro-sciences, as discussed by Intriligator (1988). Each theory contains a number of variables $x_{n}(n=1, \ldots, N)$ connected 
by a number of relations, or equations; at time $t$ a change $\Delta x_{n}$ can be forecast with the aid of these relations. The value $\Delta x_{n}$ can be the sum of $\Delta x_{n}^{l}(l$ $=1, \ldots, L)$ where $l$ stands for a science. For example $x_{n}$ is the quantity of wheat harvested and among the $l s$ is chemistry (quantity of fertilizer used), meteorology (quantities of rain fallen in various time periods, temperature in various time periods preceding harvesting), economics (price of wheat, of fertilizer, etc., technology). Forecast $\Delta x_{n}$ depends not only on the economic forecast of the farmers' reaction to price changes of wheat and fertilizer, but also on the meteorological forecasts of rain and temperature and the chemistry's forecast of the impact of fertilizer on harvest, etc. Forecasts $\Delta x_{n}^{l}$ can bc estimated later, after all forecast variables have taken place and been collected and the forecasts have been compared with reality. Among economists it is in particular Henri Theil (1958, 1966) who, more than twenty-five years ago, made important contributions to this branch of econometrics.

It is interesting to make a comparative study of many macro-sciences; for instance, in what degree they differ with respect to forecasting capability and, more particularly, with respect to their dependence of other macro-sciences. Generally speaking, natural sciences do not depend on social sciences whereas social sciences depend significantly on natural sciences. Most 'laws' of physics, whether concerning the theories of light and of sound, or the theory of gravity, cannot be influenced by economic variables. The same applies to the biological sciences. Economic variables may influence physical variables, such as those describing the human environment; but they do not influence the interrelations between physical variables. A trace of economic influences on biological variables may be found among medical activities. It is conceivable that governmental measures may have an impact on hospital equipment and so contribute to the health of a population. But again: do these measures influence the relations between biological variables; for instance, between the health of one person and another (as with contageous diseases)? A clearer impact of economics exists on applied natural sciences or technology.

Social sciences also clearly are interdependent among themselves. Economic science depends on sociology and sociology on economics. Psychology and economics are interdependent: economic variables, such as the prices of shares, clearly depend on psychological variables, such as the optimistic or pessimistic moods of dealers. Behaviour of youngsters clearly depends on the way they were educated by their parents, teachers or friends. An interesting ficld of rescarch may result from this recognition: a more precise analysis of this interconnection between (micro)sciences.

\section{References}

Intriligator, M.D., 1988, "Interdependence among the behavioural sciences", in: M. Dogan, ed., Papers at the Frontiers of the Social Sciences: Creative Marginality, forthcoming.

Nordhaus, W.D., 1987, "Forecasting efficiency: Concepts and applications", Review of Economics and Statistics, 69, pp. $667-674$.

Theil, H. 1958, Economic Forecasts and Policy (North Holland, Amsterdam).

Theil, H., 1966, Applied Economic Forecasting (North Holland, Amsterdam).

Biography: Jan TINBERGEN (1903) received a Ph.D. in Natural Science from Leyden University in 1929. His thesis supervisor was Professor Paul Ehrenfest. He has done pioneering work in Economic and Econometric Sciences. His research interests cover problems in long and short-term economic cycles, development economics, economic policy, economic modelling and income distribution. His main work is his famous book "Economic Policy: Principles and Design". He received many Honorary Doctorates. In addition he has won numerous prizes and awards, the foremost among them being the first Alfred Nobel Memorial Prize in Economic Science which he shared with Ragnar Frisch. 